\title{
ARTICLE \\ Pharmacological modulation of AMPA receptor rescues social impairments in animal models of autism
}

\author{
Ji-Woon Kim ${ }^{1}$, Kwanghoon Park ${ }^{2}$, Ri Jin Kang ${ }^{1}$, Edson Luck T. Gonzales ${ }^{1}$, Do Gyeong Kim ${ }^{1}$, Hyun Ah Oh ${ }^{1}$, Hana Seung ${ }^{1}$, Mee Jung Ko (D)
} Kyoung Ja Kwon ${ }^{3}$, Ki Chan Kim ${ }^{1}$, Sung Hoon Lee ${ }^{4}$, ChiHye Chung (iD) ${ }^{2}$ and Chan Young Shin (D)

\begin{abstract}
Autism spectrum disorder (ASD) is a neurodevelopmental disorder, featuring social communication deficit and repetitive/restricted behaviors as common symptoms. Its prevalence has continuously increased, but, till now, there are no therapeutic approaches to relieve the core symptoms, particularly social deficit. In previous studies, abnormal function of the glutamatergic neural system has been proposed as a critical mediator and therapeutic target of ASD-associated symptoms. Here, we investigated the possible roles of a-amino-3-hydroxy-5-methyl-4-isoxazolepropionic acid receptors (AMPARs) in autism symptoms using two well-known autistic animal models, Cntnap2 knockout (KO) mice and in utero valproic acid-exposed ICR (VPA) mice. We found that Cntnap2 KO mice displayed decreased glutamate receptor expression and transmission. Contrarily, VPA mice exhibited increased glutamate receptor expression and transmission. Next, we investigated whether AMPAR modulators (positive-allosteric-modulator for Cntnap2 KO mice and antagonist for VPA mice) can improve autistic symptoms by normalizing the aberrant excitatory transmission in the respective animal models. Interestingly, the AMPAR modulation specifically ameliorated social deficits in both animal models. These results indicated that AMPAR-derived excitatory neural transmission changes can affect normal social behavior. To validate this, we injected an AMPAR agonist or antagonist in control ICR mice and, interestingly, these treatments impaired only the social behavior, without affecting the repetitive and hyperactive behaviors. Collectively, these results provide insight into the role of AMPARs in the underlying pathophysiological mechanisms of ASD, and demonstrate that modulation of AMPAR can be a potential target for the treatment of social behavior deficits associated with ASD.
\end{abstract}

Neuropsychopharmacology (2019) 44:314-323; https://doi.org/10.1038/s41386-018-0098-5

\section{INTRODUCTION}

Autism spectrum disorder (ASD) is a complex neurodevelopmental disorder characterized by the two prominent behavioral symptoms of impaired social communication and restricted/repetitive behaviors. The global prevalence of ASD is increasing, and currently reaches almost $1 \%$ [1]. Its pathophysiological mechanisms are so complex that individual ASD animal models encapsulate different behavioral and pathophysiological phenotypes [2-5], which makes it difficult for the overall drug development process to succeed. Thus, it might be necessary to subdivide ASD into more specific groups based on an understanding of the pathophysiological mechanism.

One possible criterion for subdividing ASD is the excitatory-inhibitory imbalance (E/I imbalance) hypothesis [6]. Most neurons in the brain are connected to each other through excitatory or inhibitory synapses. Any structural or functional abnormality in these synapses can cause misprocessing of information and connectivity problems (reviewed in refs. [5, 6]). Indeed, a previous optogenetic study demonstrated that abnormal activation of the excitatory neuron in the frontal cortex causes social impairments and abnormal electroencephalography (EEG) synchrony [7]. Additionally, patients with ASD, as well as autistic animal models, display abnormal expression of synaptic proteins and functional defects in their excitatory or inhibitory neurons, which has led to the $E / I$ imbalance hypothesis being a major approach to understanding the pathophysiology of ASD [5, 8-11].

On the basis of the E/I imbalance hypothesis, glutamatergic receptors, especially, $\mathrm{N}$-methyl-D-aspartate receptors (NMDARs) have been regarded as a potential therapeutic target to relieve autism-like symptoms in preclinical studies [12-15]. However, a large number of clinical or preclinical studies also suggest that $a-$ amino-3-hydroxy-5-methyl-4-isoxazolepropionic acid receptors (AMPARs) may be implicated in ASD. In a postmortem study, increased mRNA and protein expression levels of GluA1 were observed in the cerebellum of patients with ASD [16]. In clinical genetic studies, copy number variations have been reported in the genetic loci of AMPAR, and deletion of GRIA2 gene was also found in patients with ASD [17-20]. In preclinical studies, Gria1 knockout (KO) mice exhibited decreased social interactions [21]. Further, ASD mouse models, such as $N / g n 3^{R 704 C}$ knock-in mice and Ube3a KO mice, displayed aberrant AMPAR subunit expression and functional impairment of AMPARs in the

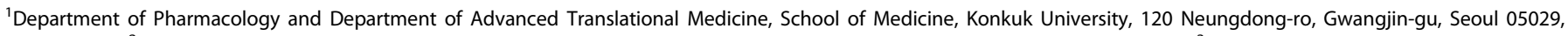

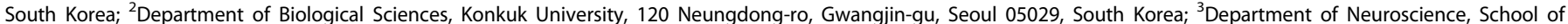

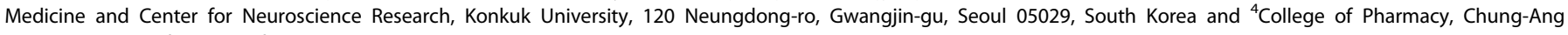
University, Dongjak-gu, Seoul 06974, Korea

Correspondence: Chan Young Shin (chanyshin@kku.ac.kr)

These authors contributed equally: Ji-Woon Kim, Kwanghoon Park.

Received: 11 October 2017 Revised: 9 May 2018 Accepted: 14 May 2018

Published online: 22 May 2018 
hippocampus $[22,23]$. These results prompted us to consider a role of AMPARs in ASD.

Here, to investigate whether AMPARs can be a potential therapeutic target, we used two animal models: a contactinassociated protein-like 2 gene (Cntnap2) KO mouse model, and a prenatal exposure of valproic acid-induced mouse model (VPA mice). CNTNAP2 is a well-established ASD risk factor, and its mutation is frequently observed in patients with ASD [24, 25]. Cntnap2 KO mice are a validated ASD animal model exhibiting repetitive grooming behaviors and social deficits [26]. In utero VPA exposure is a well-known environmental risk factor for ASD, and increases the occurrence rate by $\sim 20$ times [27]. The VPA mice display autism-like behaviors, such as the social deficit, repetitive grooming, and hyperactive behavior, $[28,29]$ and the glutamatergic transmission in their prefrontal cortex (PFC) was found to be enhanced $[13,30]$.

In this study, we found that Cntnap2 KO and VPA mice displayed reduced and increased glutamatergic receptor expression and function, respectively, although their autism-like behaviors were similar. Interestingly, administration of AMPAR agonist or antagonist to Cntnap2 KO or VPA mice rescued the social deficits, but not other ASD-like behaviors. On the basis of these findings, we postulated that social behaviors can be affected by AMPAR activity, and administered AMPAR agonist or antagonist to control Istitute of Cancer Research (ICR, Hsd:ICR or CD-1) mice, and monitored their behavioral changes. Remarkably, either of the AMPAR agonist or antagonist treatment specifically impaired normal social behaviors of the control mice. Thus, our results suggest that the regulation of excitatory neural activity by AMPARs is crucial for normal social behaviors, suggesting the importance of AMPAR transmission in the pathophysiological mechanism of ASD.

\section{MATERIALS AND METHODS}

Animals

Cntnap2-KO mice were a generous gift from Dr. Daniel $\mathrm{H}$. Geschwind [26]. For the VPA-induced animal model of autism (VPA-exposed mice or VPA mice), prenatal exposure of VPA or PBS was performed as previously reported [28]. Mice were injected with $300 \mathrm{mg} / \mathrm{kg}$ of VPA subcutaneously at embryonic day 10 . The maintenance conditions and ethics protocol have been described in the supplementary information.

\section{Drug treatment}

We intraperitoneally injected CP465022 $(0.25,0.5$, and $1 \mathrm{mg} / \mathrm{kg}$, AMPAR antagonist), PF4778574 (0.15 and $0.3 \mathrm{mg} / \mathrm{kg}$, positiveallosteric modulator [PAM]) or vehicle (5\% DMSO in saline) $30 \mathrm{~min}$ before each behavioral experiment. These drugs have been reported to be AMPAR-selective and brain-penetrable, and dosages and route of administration were selected based on previous studies and preliminary tests [31, 32].

Three-chamber social interaction test

The three-chamber social interaction test was performed from P28 to P32 as previously reported [28]. After habituation for $5 \mathrm{~min}$ in the central compartment of the arena, a novel conspecific mouse was introduced into the wire cage of either the left or right compartment (stranger zone 1) randomly for the sociability test. Subsequently, social preference test was conducted for 10 min. A new conspecific mouse was introduced into the wire cage of the opposite compartment (stranger zone 2), followed by measurement of time spent in each compartment, as in the previous session. Time spent in each compartment and near each cage (5$\mathrm{cm}$ distance from the cage) was measured as stay duration and approach duration, respectively (Supplementary Figure 9). The traces of the mouse movements during the experiments were automatically recorded using the EthoVision software.
Juvenile social play

The test was performed from P24 to P26, as previously described, with a slight modification [28]. Before the test, the subject mouse was isolated for $30 \mathrm{~min}$ in a new cage with clean bedding. Subsequently, a conspecific stranger mouse of the same age and sex as the subject was introduced into the cage. Accumulated social interaction time, including sniffing, following, allogrooming, and crawling under the partner, was measured for $10 \mathrm{~min}$.

\section{Repetitive self-grooming test}

The test was performed from P30 to P35 as previously reported [28]. Before the experiment, each mouse was placed in the polycarbonate cage without bedding to stimulate grooming behavior, and was habituated for $10 \mathrm{~min}$. The cumulative grooming time was measured for another $10 \mathrm{~min}$ by a "blind observer" $2 \mathrm{~m}$ away from the cage.

\section{Western blot analysis}

Brain tissues were collected at P28. The tissues were homogenized using RIPA buffer (150 mM sodium chloride, $1 \%$ Triton X-100, $0.5 \%$ sodium deoxycholate, $0.1 \% \mathrm{SDS}, 50 \mathrm{mM}$ Tris, $\mathrm{pH} 8.0$ ) with protease and phosphatase inhibitors (protease inhibitor cocktail [Roche, No.11873580001], $50 \mathrm{mM} \mathrm{NaF}, 1 \mathrm{mM}$ PMSF, and $1 \mathrm{mM} \mathrm{Na} \mathrm{VO}_{4}$ ). BCA was performed to quantify the protein concentrations, and equal amounts of proteins were loaded on $8 \%$ SDS-PAGE gels, and then transferred to nitrocellulose membranes. After transfer, membranes were incubated with $1 \%$ skim milk for $1 \mathrm{~h}$, and with primary antibody overnight at $4{ }^{\circ} \mathrm{C}$. A 2-h incubation with the peroxidase-conjugated secondary antibody (Invitrogen; rabbit: G21040, mouse: G21234) at room temperature followed, and the signals of the blots were detected using the WEST-ZOL Plus (iNtRON Biotechnology, 16024), and exposed to LAS-3000 imaging system (Fuji film). The blots were quantified using multi-gauge V3.0 software (Fuji film), and normalized with $\beta$-actin immunoreactivity.

\section{Slice preparation}

Slices were prepared from P28 to P35 mice. Before decapitation, mice were anesthetized with isoflurane, and the brains were rapidly collected and transferred into ice-cold sucrose dissecting solution containing (in $\mathrm{mM}$ ) 212 sucrose, $3 \mathrm{KCl}, 26 \mathrm{NaHCO}_{3}, 1.25$ $\mathrm{NaH}_{2} \mathrm{PO}_{4}, 7 \mathrm{MgCl}_{2}$, and 10 glucose. Coronal slices (350- $\mu \mathrm{m}$-thick) containing the medial PFC ( $\mathrm{mPFC}$ ) were obtained using a Leica VT1200S vibratome. Slices were stored in a submerged holding chamber filled with artificial cerebrospinal fluid containing (in $\mathrm{mM}$ ) $118 \mathrm{NaCl}, 2.5 \mathrm{KCl}, 1 \mathrm{NaH}_{2} \mathrm{PO}_{4}, 26.2 \mathrm{NaHCO}_{3}, 11$ glucose, $2 \mathrm{CaCl}_{2}$, and $1 \mathrm{MgCl}_{2}$ (oxygenated with $95 \% \mathrm{O}_{2} / 5 \% \mathrm{CO}_{2}$ ) at $35^{\circ} \mathrm{C}$. After $1 \mathrm{~h}$ of recovery, brain slices were kept at room temperature during recording.

\section{Electrophysiology}

Neurons in the mPFC were voltage clamped at -60 or $0 \mathrm{mV}$ to measure the excitatory or inhibitory transmission, respectively, using an Axoclamp-200B amplifier (Axon Instruments, Union City, (A, USA), filtered at $2 \mathrm{kHz}$ and sampled at $5 \mathrm{kHz}$. The pClamp software (Version 10.3, Axon Instruments) was used for data acquisition and analysis. The resistance of patch glass pipettes was 2-4 M $\Omega$, and the internal solution contained (in $\mathrm{mM}$ ) $115 \mathrm{Cs}$ methanesulfonate, $20 \mathrm{CsCl}, 10$ HEPES, $2.5 \mathrm{MgCl}_{2}, 4 \mathrm{Na}_{2}$-ATP, 0.4 $\mathrm{Na}_{2}$-GTP, 10 Na-phosphocreatine, and 0.6 EGTA (pH 7.2). To measure the miniature excitatory postsynaptic currents (mEPSCs), picrotoxin $(50 \mu \mathrm{M})$ and tetrodotoxin $(1 \mu \mathrm{M})$ were added throughout the experiments. To measure the miniature inhibitory postsynaptic currents (mIPSCs), CNQX (10 $\mu \mathrm{M})$, AP5 (50 $\mu \mathrm{M})$, and tetrodotoxin $(1 \mu \mathrm{M})$ were added throughout the experiments. Input and series resistances were continuously monitored. 


\section{RESULTS}

Cntnap2 KO mice display reduced glutamate receptor expression and function

We analyzed the expression of AMPAR subunits, GluA1 and GluA2, and NMDAR subunits, GluN1, GluN2A, and GluN2B, in the PFC (Fig. 1a, b). Interestingly, GluA1, GluA2, GluN2A, and GluN2B, but not GluN1, expressions were reduced in Cntnap2 KO mice. We also confirmed these results using primary cortical neuronal cultures (Supplementary Figure 1). We infected primary neurons with lentiviral Cntnap2 shRNA knockdown (KD) construct at days in vitro (DIV) 7, and measured the glutamate receptor expression at DIV12. Consistent with the in vivo results, reduced expressions of GluA1, GluA2, GluN2A, and GluN2B were observed in Cntnap2 KD-cortical neurons, suggesting that reduced CNTNAP2 expression ( $50 \%)$ during neural development affects the expression of glutamate receptors. Next, to determine whether reduced glutamate receptor expression affects synaptic transmission, we measured the mEPSC amplitude and frequency in the mPFC (infralimbic cortex, layer $2 / 3$ pyramidal neurons) using a wholecell patch clamp (Fig. 1c-e). The amplitude was significantly reduced in Cntnap2 $\mathrm{KO}$ mice, indicating reduced function or number of postsynaptic glutamate receptors, while the frequency, an indicator of presynaptic function, remained unchanged.

\section{AMPAR agonist rescues impaired social behavior of Cntnap2 KO} mice

To determine the role of AMPARs in the autism-like behavioral phenotypes of Cntnap2 KO mice, we performed the threechamber social interaction test (Fig. 2a-d) and repetitive selfgrooming test (Fig. 2e), 30 min after administration of the AMPAR agonist PF4778574. Vehicle-treated Cntnap2 KO mice did not show preference to the compartment with a conspecific stranger mouse, but treatment with PF4778574 in these mice rescued this impaired social behavior (Fig. 2a). We also analyzed the approach duration, representing the stay duration near the cage in each compartment. Similarly, PF4778574 treatment significantly restored the impaired social behavior in Cntnap2 KO mice (Fig. 2b). In addition, Cntnap2 KO mice displayed reduced preference to a novel mouse in the social preference test, which was also rescued by PF4778574 treatment (Fig. 2c, d). However, AMPAR agonist treatment did not significantly ameliorate the excessive repetitive self-grooming behavior of Cntnap2 KO mice, although it showed decreasing trends (Fig. 2e). To verify that the therapeutic effect was not age-dependent, we confirmed the impaired social behavior and repetitive grooming in adult mice (9-12 weeks old, Supplementary Figure 2). Consistent with the findings in young mice, social impairments, but not repetitive self-grooming behaviors, were rescued by PF4778574 treatment in adult Cntnap2 KO mice. Interestingly, PF4778574 treatment of wild-type (WT) mice induced social impairment not only in the sociability test but also in the social preference test (Fig. 2a-d), suggesting that excessively increased neural transmission due to AMPAR agonist treatment might lead to abnormal social behaviors.

Expression of ionotropic glutamate receptors and excitatory synaptic transmission are increased in in utero VPA-exposed mice In previous studies, in utero VPA-exposed rats displayed increased expression levels of glutamate receptors and enhanced excitatory synaptic transmission, especially in the PFC $[13,33]$. To validate these observations in our mouse model, we performed western blotting using the PFC tissue from these mice (Fig. 3a, b). We found that the expression levels of GluA1 and GluN2B were significantly increased in the VPA mice. Moreover, our whole-cell patch clamp analysis revealed that the amplitude of mEPSCs in the mPFC of VPA-exposed mice was significantly increased compared to that of control mice, while the frequency remained unchanged (Fig. 3c-e). When we analyzed our whole-cell patch clamp data in a layer-specific manner (Supplementary Figure 3), the pyramidal neurons in the superficial layer (layer 2/3) of VPA-exposed mice displayed significantly larger amplitude of mEPSCs than that of control mice, while the amplitude of mEPSCs in the deep layer (layer 5) of VPA-exposed mice was not different from that of control mice. There was no layer-dependent difference in the mEPSCs frequency between groups. We also measured the mIPSCs in the MPFC, but there were no significant changes in the amplitude and frequency of mIPSCs (Supplementary Figure 4).

Suppression of AMPAR-mediated transmission rescues social deficits in in utero VPA-exposed mice

To investigate the effect of AMPAR activity suppression on autismlike behaviors of VPA mice, an AMPAR antagonist, CP465022, was administered intraperitoneally, and behavioral tests were performed 30 min later (Fig. 4 and Supplementary Figure 5). In the sociability test, VPA mice did not stay significantly longer in the conspecific stranger mouse area than the empty cage area (Fig. 4a, b). This impaired social behavior was rescued by $\mathrm{CP} 465022$ treatment at the doses of 0.5 and $1 \mathrm{mg} / \mathrm{kg}$. In the social preference test, VPA-exposed mice did not exhibit a significant preference to the novel mouse (Fig. 4c, d). The impaired social preference was also rescued by CP465022 treatment at all doses. The therapeutic effects of CP465022 on social behaviors were re-evaluated in the juvenile social play test (Fig. 4e). The cumulative social behavior time significantly increased upon treatment with 0.5 and $1 \mathrm{mg} / \mathrm{kg}$ of CP465022, confirming the above results. In the grooming test, VPA-exposed mice exhibited increased grooming time, which was not rescued by CP465022 treatment (Fig. 4f). We also measured the repetitive digging behavior, but CP465022 treatment failed to reduce the excessive digging behavior of VPA mice (Supplementary Figure $5 \mathrm{a}$ ). In the open field test, the increased distance moved and stay duration within the center area of VPA mice was not rescued by treatment with 0.25 and $0.5 \mathrm{mg} / \mathrm{kg} \mathrm{CP465022} \mathrm{but,}$ at $1 \mathrm{mg} / \mathrm{kg}, \mathrm{CP} 465022$ slightly reduced the increased distance moved and stay duration within the center area (Supplementary Figure $5 b$ and $c$ ).

Dysregulated AMPAR activity diminishes the normal social behaviors of control ICR mice

Our results suggested that modulation of AMPAR activity can correct abnormal social behavior, possibly by correcting the aberrant excitatory neural transmission. On the basis of these results we hypothesized that dysregulation of AMPAR-derived excitatory neural transmission can induce abnormal social behaviors. To investigate this hypothesis, we administered either AMPAR agonist (PF4778574) or antagonist (CP465022) to control ICR mice, and investigated their behavioral changes (Fig. 5). The AMPAR agonist, PF4778574, at $0.3 \mathrm{mg} / \mathrm{kg}$ which is a high dosage, reduced the time spent in the stranger mouse area during the sociability test (Fig. 5a, b). This dosage only slightly affected the social novelty preference, but the same was not observed for lower doses (Fig. 5c, d). Interestingly, we also confirmed the impaired social behavior induced by AMPAR agonist in the juvenile social play test, where the social interaction was significantly decreased in the $0.3 \mathrm{mg} / \mathrm{kg}$ dose group (Supplementary Figure 6a).

On the other hand, the group treated with the high dosage of AMPAR agonist $(1 \mathrm{mg} / \mathrm{kg}$ ) also displayed reduced preference to the peer mouse over the empty cage during the sociability test (Fig. 5h, i). However, the preference to the novel mouse was intact in all dosage groups during the social preference test (Fig. 5j, k). Interestingly, neither AMPAR agonist nor antagonist treatment affected the repetitive behavior at any dose tested. We also confirmed that these social behavioral impairments were not due to abnormal locomotor activity, or anxiety or spatial memory problems using the open field and Y-maze tests after AMPAR agonist or antagonist treatment (Supplementary Figures 6 and 7). 
a

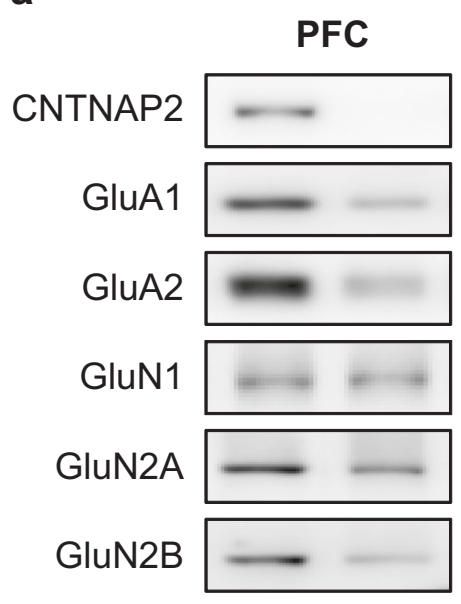

actin

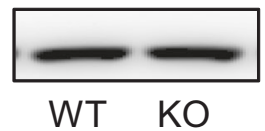

b
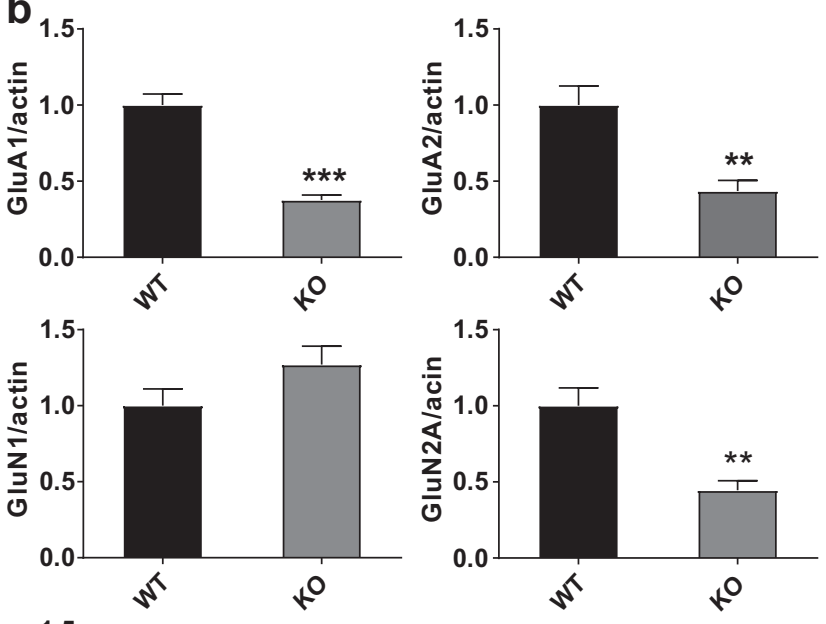

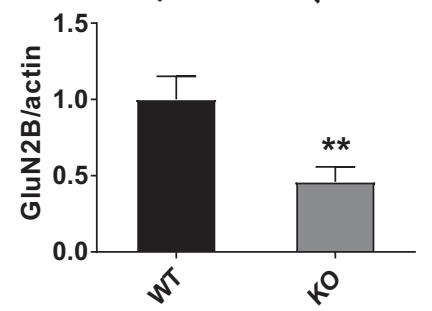

C MPFC
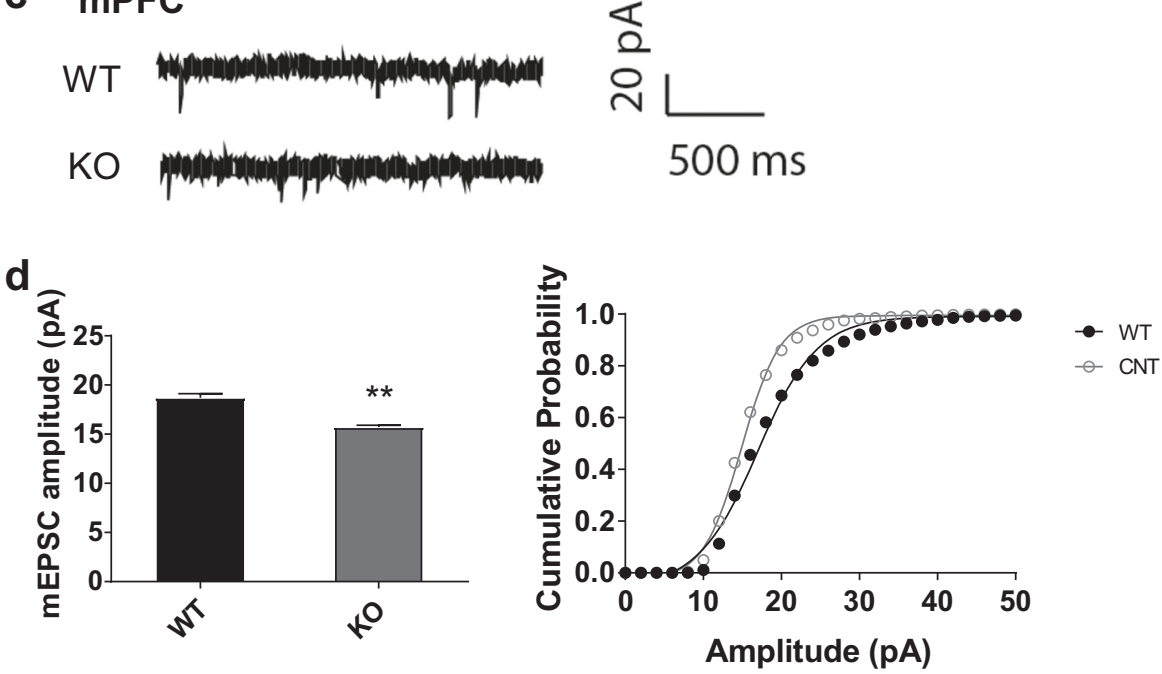

e
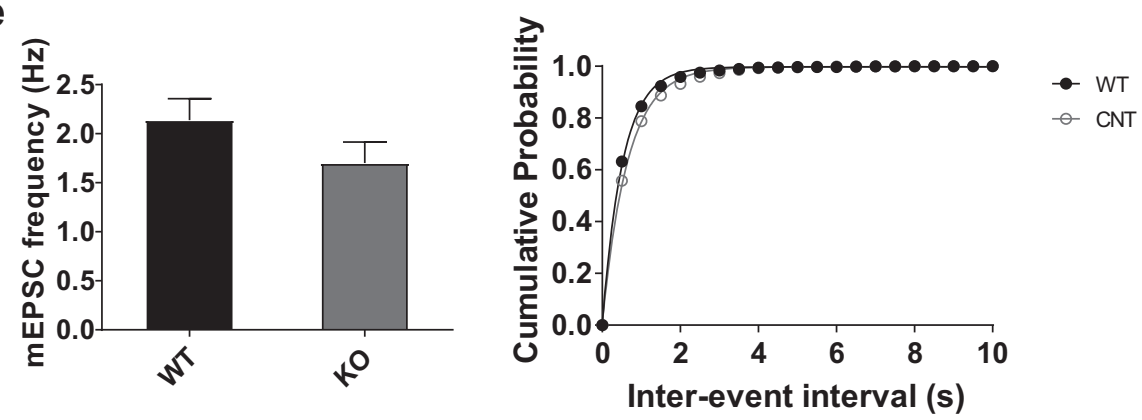

Fig. 1 Decreased expression of ionotropic glutamate receptors and reduced excitatory synaptic transmission in Cntnap2 KO mice. To quantify the expression level of proteins and measure synaptic transmission western blotting $(\mathbf{a}, \mathbf{b})$ and whole-cell patch clamp recordings $(\mathbf{c}-\mathbf{e})$ were performed, respectively, using the medial prefrontal cortex. a, b The levels of ionotropic glutamate receptor subunits GluA1, GluA2, GluN2A, and GluN2B, but not GluN1, were significantly decreased in Cntnap2 knockout (KO) mice. c Representative traces of the miniature excitatory postsynaptic currents (mEPSCs). d, e The amplitude of mEPSCs, but not frequency, was significantly decreased in Cntnap2 KO mice. For western blotting: $N=8-10$, and for electrophysiology: $N=\mathrm{WT}: 18, \mathrm{KO}: 17$. ${ }^{* * * * *} p<0.01,0.001$, respectively vs. Con. In the graph, data are expressed as the mean \pm standard error of the mean 

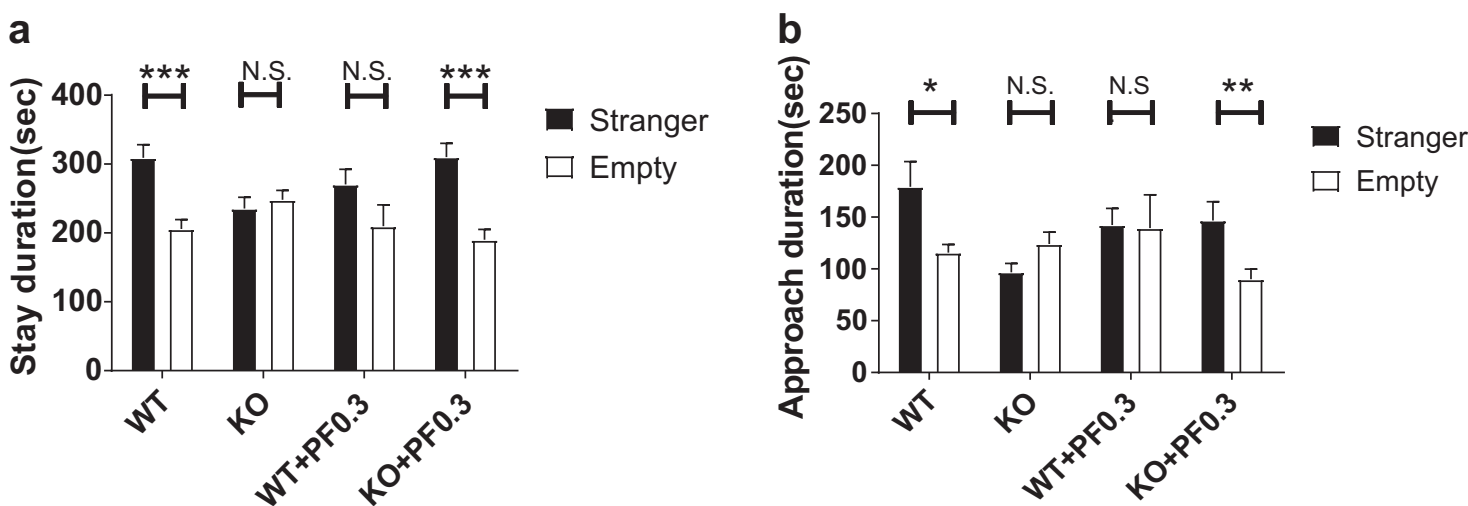

Preference
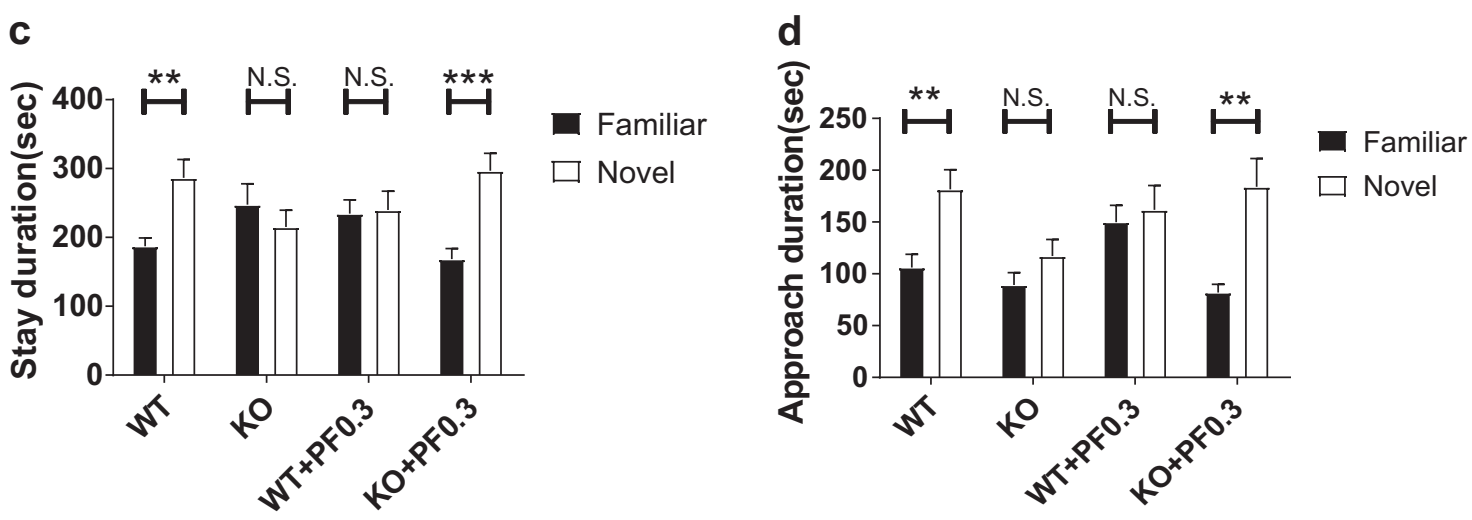

e Grooming test

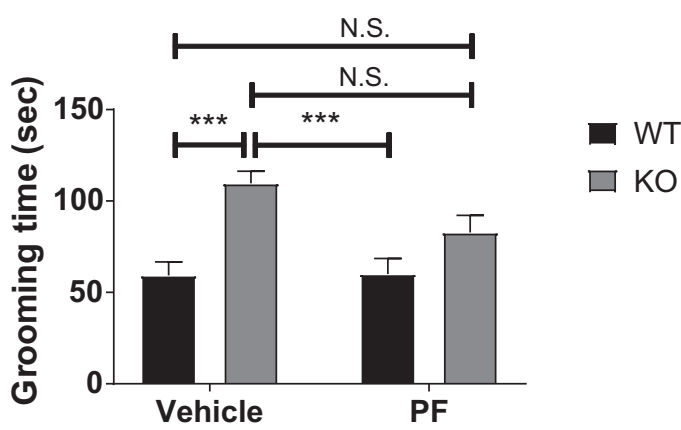

Fig. 2 Activation of AMPAR rescues social deficit in Cntnap2 KO mice. After intraperitoneal administration of $\alpha$-amino-3-hydroxy-5-methyl-4isoxazolepropionic acid receptor (AMPAR) agonist, PF4778574, in Cntnap2 knockout (KO) mice, three-chamber social interaction test (a-d) or grooming test (e) was performed. In the three-chamber social interaction test, the stay duration (a, c), and approach duration (b, d) were measured. The impaired sociability and social preference behavior in Cntnap2 KO mice were improved by PF4778574 treatment (stranger vs. empty or familiar vs. novel; N.S. not significant, $*_{*}^{* * * * *} p<0.05,0.01,0.001$, respectively; WT: 12, KO: 13 , WT + PF0.3: 9, KO + PF0.3: 10 ). e Grooming test. The increased repetitive grooming behavior in Cntnap2 KO mice was not significantly reduced by PF4778574 treatment but showed only a slight decreasing trend; WT: 9, KO: 9, WT + PF0.3: 9, KO + PF0.3: 9; N.S. not significant, ${ }^{* * *} p<0.001$, respectively. In the bar graph, data are expressed as the mean \pm standard error of the mean (WT: Cntnap2 wild-type mice, KO: Cntnap2 KO mice, PF0.3: PF4778574, 0.3 $\mathrm{mg} / \mathrm{kg}$ )

Thus, our results suggest that AMPAR-mediated excitatory neural activity regulation is crucial to maintain normal social behaviors.

\section{DISCUSSION}

In the present study, we found that administration of AMPAR PAM improved social deficits in Cntnap2 KO mice displaying decreased expression of glutamate receptors and reduced amplitude of mEPSCs. In contrast, AMPAR antagonist treatment rescued the social deficits in VPA mice exhibiting enhanced GluA1 and GluN2B expression and increased mEPSC amplitude. Moreover, we found that treatment with a high dose of AMPAR modulators induced social impairments without affecting repetitive behaviors, hyperactivity, and cognitive deficits in control mice. Thus, our results 
a

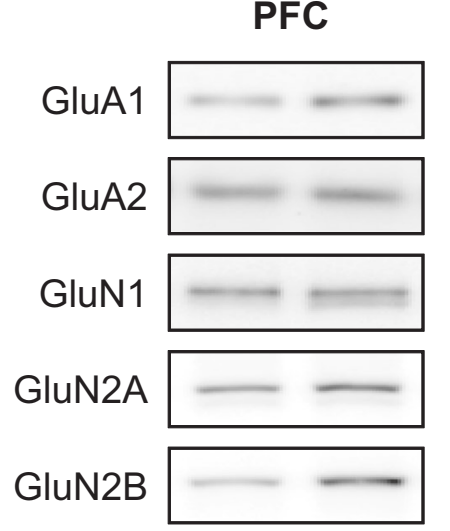

$\operatorname{actin} \longrightarrow$

Con VPA
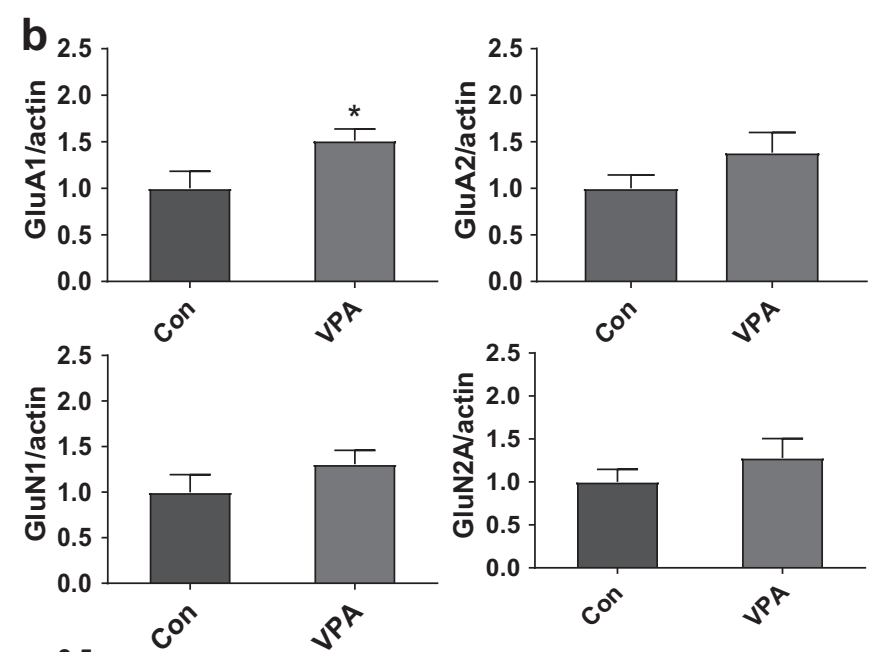

C. $\mathrm{MPFC}$
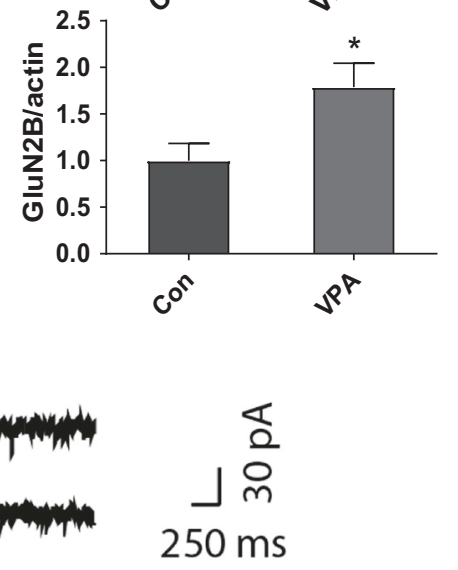

d.
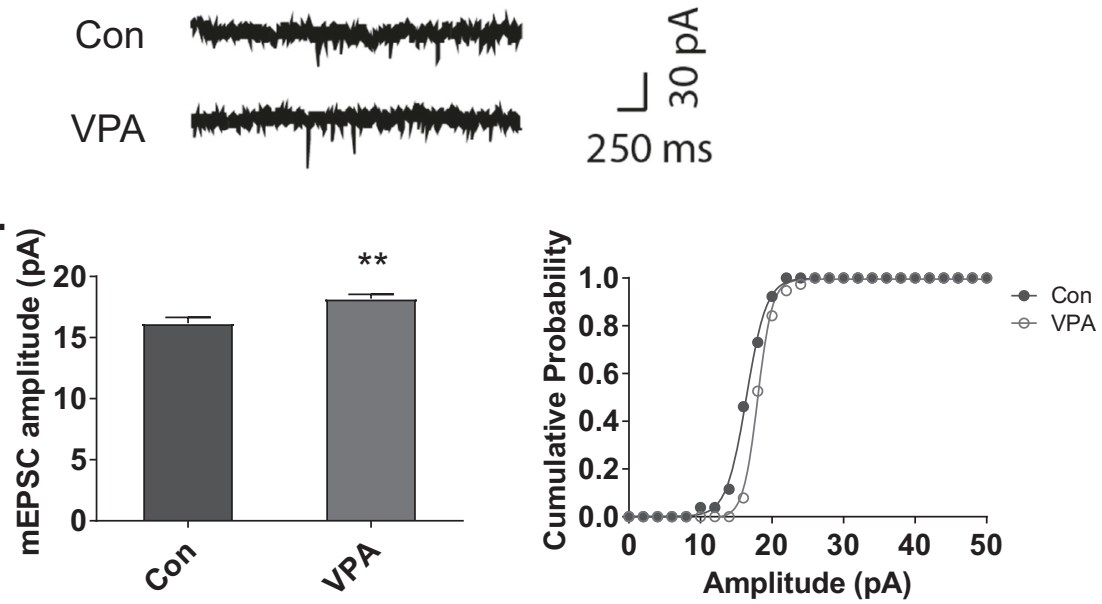

e.
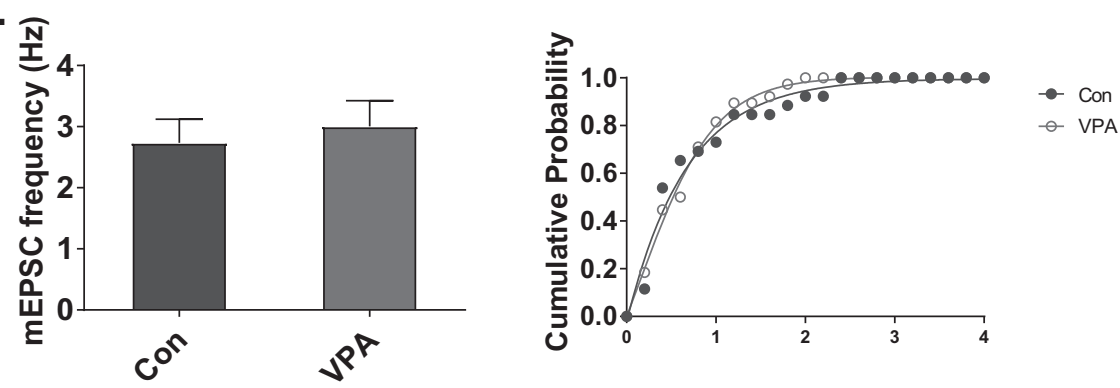

Fig. 3 Increased expression of ionotropic glutamate receptors and enhanced excitatory synaptic transmission in VPA mice. To quantify the expression level of proteins and measure the synaptic transmission, western blotting (a, b) and whole-cell patch clamp recording (c-e) were performed, respectively, using the medial prefrontal cortex (MPFC) region. $\mathbf{a}$, $\mathbf{b}$ The levels of GluA1 and GluN2B were significantly increased in VPA mice. c Representative traces of miniature excitatory postsynaptic currents (mEPSCs) in the mPFC. $\mathbf{d}$, e The amplitude of mEPSCs, but not frequency, was significantly increased in VPA mice (western blotting: $N=$ Con: 7, VPA: 7; electrophysiology: $N=$ Con: 26 , VPA: 38 ; ${ }^{*} * *<0.05$, 0.01 , respectively vs. Con). In the graph, data are expressed as the mean \pm standard error of the mean (Con: mice exposed to saline on embryonic day 10, VPA: mice exposed to valproic acid on embryonic day 10 ) 
Sociability

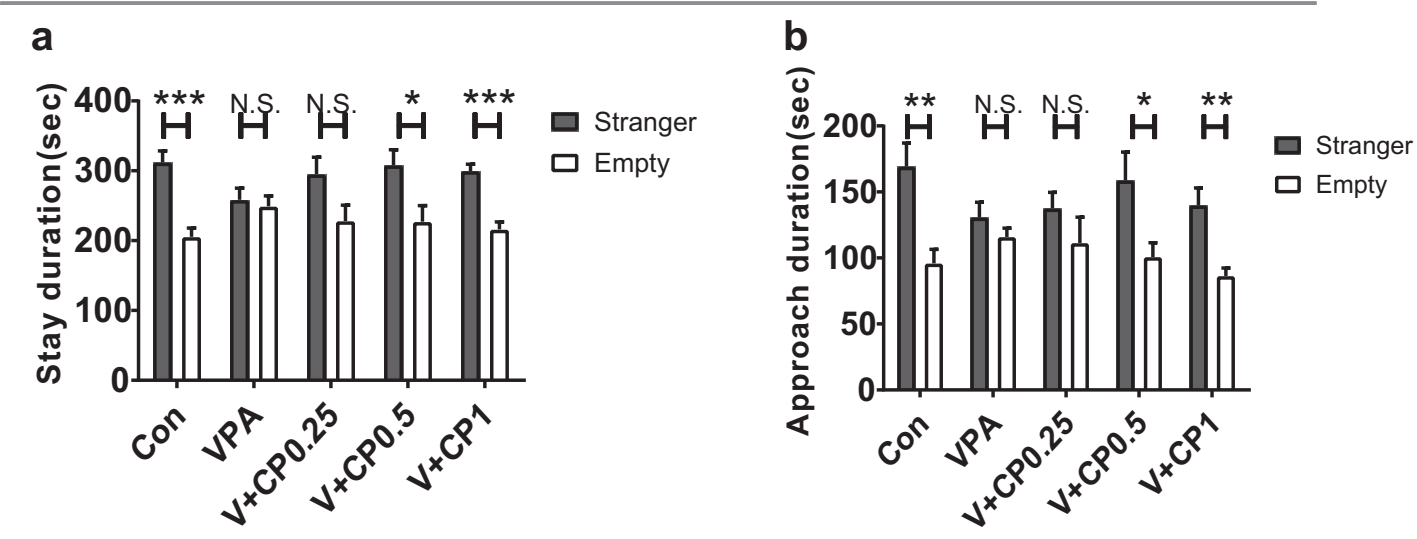

Preference
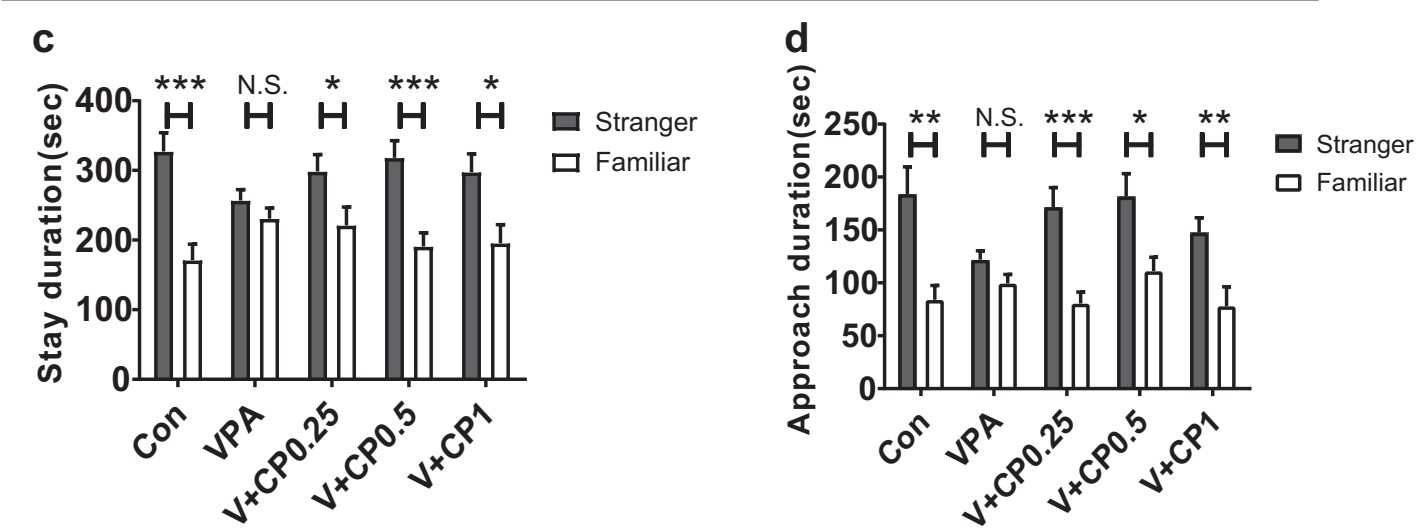

e Juvenile social play

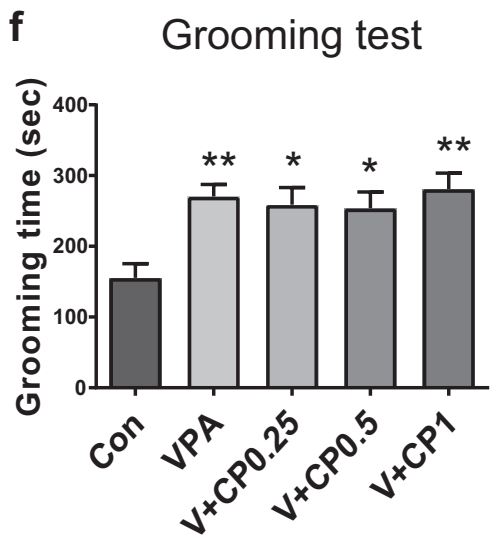

Fig. 4 Suppression of AMPAR activity rescues social deficit in VPA-exposed mice. After intraperitoneal administration of $\alpha$-amino-3-hydroxy-5methyl-4-isoxazolepropionic acid receptor (AMPAR) antagonist, CP465022, in VPA-exposed mice, three-chamber social interaction test $(\mathbf{a}-\mathbf{d})$, juvenile social play test (e), and repetitive self-grooming test (f) were performed. In the three-chamber social interaction test, the stay duration $(\mathbf{a}, \mathbf{c})$ and approach duration $(\mathbf{b}, \mathbf{d})$ were measured (N.S. not significant, ${ }^{*} * *_{*} * * * p<0.05,0.01,0.001$, respectively; 6 litters for Con, 12 litters for VPA and treatment groups; Con: 10, VPA: 13, V + CP0.25: 10, V + CP0.5: 10, V + CP1: 11). e Juvenile social play test. Cumulative social interaction time was measured for $10 \mathrm{~min}(* * p<0.01$, vs. control; $\#$, \#\#\# $p<0.05,0.001$, respectively vs. VPA; 6 litters for Con, 12 litters for VPA and treatment groups; $N=$ Con: 9, VPA: 10, V + CP0.25: 8, V + CP0.5: 9, V + CP1: 10). f Repetitive result of self-grooming test. The increased repetitive grooming was not reduced by the AMPAR antagonist treatment in VPA mice $\left({ }^{*} * * p<0.05,0.01\right.$, respectively vs Con; 3 litters for Con, 8 litters for VPA and treatment groups; $N=$ Con: 8, VPA: 8, V + CP0.25: 8, V + CP0.5: 8, V + CP1: 8). In the bar graph, data are expressed as the mean \pm standard error of the mean (Con: mice exposed to saline on embryonic day 10; VPA: mice exposed to valproic acid on embryonic day 10; CP0.25: CP465022, 0.25 mg/kg; CP0.5: CP465022, 0.5 mg/kg; CP1: CP465022, 1 mg/kg)

suggest that AMPARs-mediated excitatory transmission can regulate social behaviors, which provides clues to understand the pathophysiological mechanisms of ASD.

Given that Cntnap2 KO mice display reduced mEPSCs amplitude but not frequency (Fig. 1), and reduced expression of ionotropic glutamate receptors, CNTNAP2 seems to mediate the expression of ionotropic glutamate receptors in the postsynapse. A previous study suggested that CNTNAP2 might be involved in regulating GluA1 trafficking. In the study, hippocampal neurons derived from Cntnap2 KO mice displayed accumulation of GluA1 in the soma 

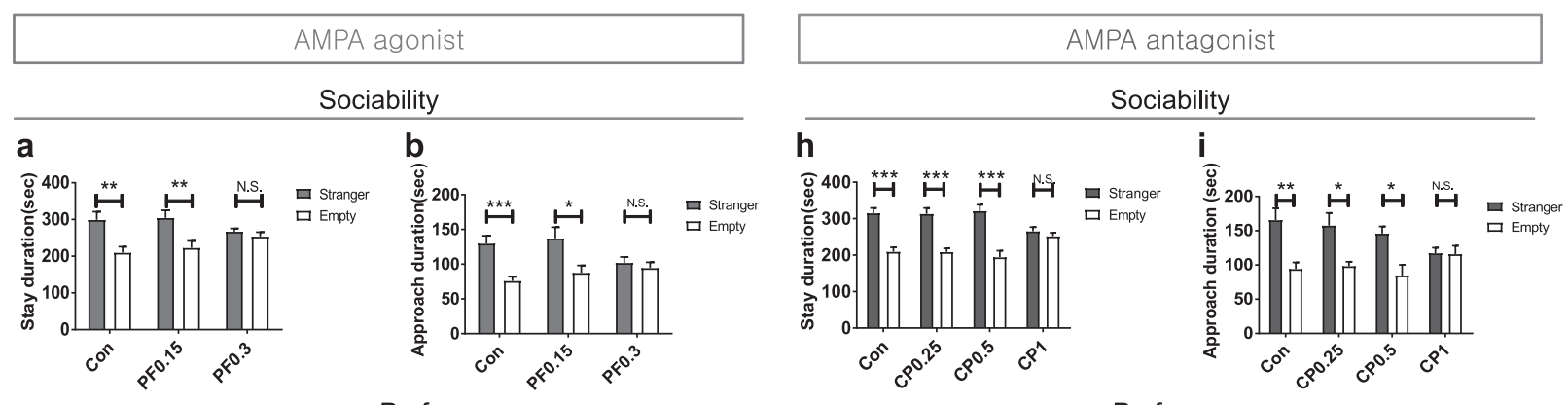

Preference
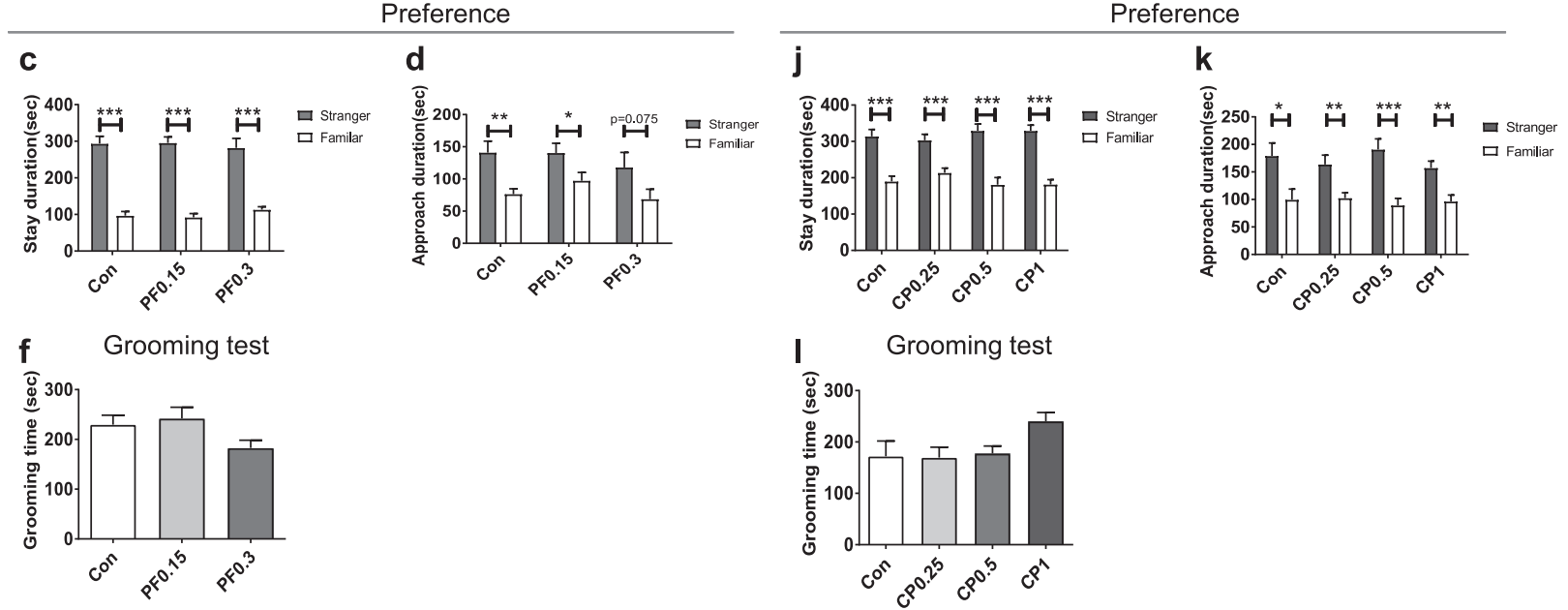

Fig. 5 Either activation or suppression of AMPAR activity affects social behavior in control ICR mice. After treatment of $\alpha$-amino-3-hydroxy-5methyl-4-isoxazolepropionic acid receptor (AMPAR) agonist, PF4778574 (a-e), or antagonist, CP465022 (h-I), in control ICR mice, threechamber social interaction test (a-d, $\mathbf{h}-\mathbf{k})$ and repetitive self-grooming test $(\mathbf{f}, \mathbf{l})$ were performed. In the three-chamber social interaction test, the stay duration $(\mathbf{a}, \mathbf{c}, \mathbf{h}, \mathbf{j})$ and approach duration $(\mathbf{b}, \mathbf{d}, \mathbf{i}, \mathbf{k})$ were measured. The $0.3 \mathrm{mg} / \mathrm{kg}$ PF4778574 treatment impaired the normal

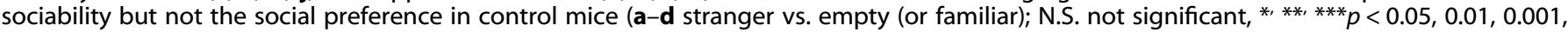
respectively; Con: 10, PF0.15: 10, PF0.3: 10). The $1 \mathrm{mg} / \mathrm{kg}$ CP465022 treatment impaired the normal social behaviors, but the social preference was not affected (h-k; N.S. not significant, $*^{* * * * *} p<0.05,0.01,0.001$, respectively; $N=$ Con: 11, CP0.25: 11, CP0.5: 11, CP1: 11 ). f, I The cumulative grooming time was measured for $10 \mathrm{~min}$ in the grooming test after the AMPAR agonist or antagonist treatment. Neither AMPAR agonist nor antagonist treatment affected repetitive self-grooming behaviors (f: $N=$ Con: 10, PF0.15: 10, PF0.3: 10; I: $N=$ Con: 10, CP0.25: 10 , CP0.5: 10, CP1: 10) In the bar graph, data are expressed as the mean \pm standard error of the mean (Con: vehicle group; PF0.15: PF4778574, 0.15 $\mathrm{mg} / \mathrm{kg}$; PF0.3: PF4778574, $0.3 \mathrm{mg} / \mathrm{kg}$; PF4778574: AMPAR agonist; CP0.25: CP465022, 0.25 mg/kg; CP0.5: CP465022, 0.5 mg/kg; CP1: CP465022, $1 \mathrm{mg} / \mathrm{kg}$; CP465022: AMPAR antagonist)

and reduced expression on the synaptic surface [34]. However, our whole-cell lysate blots showed that, in addition to GluA1, other ionotropic glutamate receptors were also reduced in Cntnap2 KO mice (Fig. 1). Thus, it may be more plausible that CNTNAP2 is involved in maintaining synaptic stability or synaptic reorganization. Indeed, Cntnap2 shRNA KD in cortical neurons reduced EPSCs charge transfer without affecting the burst frequency, reduced spine density, and reduced neurite branches. However, these impairments were restored by the recovery of Cntnap2 expression [35]. Moreover, Cntnap2 KO mice showed reduced stability of newly formed spines in layer 5 pyramidal neurons of the somatosensory cortex and reduced spine density [36]. Although we could not explore this possibility here, our future study will address the role of CNTNAP2 in maintaining synaptic stability.

The VPA mice exhibited increased excitatory transmission and ionotropic glutamate receptor expression, consistent with previous results $[13,30,33,37]$. In our previous studies, we showed that prenatal exposure to VPA induced increased glutamatergic neuronal differentiation, which might lead to enhanced excitatory synaptic function in this animal model $[13,37]$.

Although we could not demonstrate neural activity changes in the living animal after treatment with AMPAR modulators, the previously demonstrated pharmacokinetic and pharmacodynamics properties of PF4778574 and CP465022 support our findings. CP465022 is a highly selective, noncompetitive AMPAR antagonist [38], and it blocks AMPAR currents regardless of the stimulation and voltage clamping ( -60 and $+30 \mathrm{mV})$ [38] by preventing AMPAR channel opening [39]. Additionally, subcutaneous or intravenous injection of CP465022 reduced the population peak amplitude in the hippocampus [31], suggesting that peripheral administration of CP465022 can reduce excitatory neural activity in the brain. On the other hand, PF4778574 is a brain-penetrable AMPAR PAM. Subcutaneous injection of PF4778574 $(0.178 \mathrm{mg} / \mathrm{kg})$ led to a concentration in the brain of approximately $1.52 \mathrm{nM}$. In cortical neurons, PF4778574 activated AMPAR currents at a concentration higher than $3 \mathrm{nM}$ [32]. To demonstrate its effectiveness in the brain directly, we confirmed the effects of PF4778574 using whole-cell patch clamp in layer $2 / 3$ pyramidal neurons of the MPFC (Supplementary Figure 8). We administered PF4778574 at two different concentrations (1 and $10 \mu \mathrm{M})$, and confirmed that $1 \mu \mathrm{M}$ of PF4778574 was sufficient to increase the mEPSC amplitude to the maximum, while the mEPSC frequency was unaffected in Cntnap2 WT mice, indicating that the drug treatment did not affect the presynaptic function. In addition, the reduced mEPSC amplitude in Cntnap2 KO mice was enhanced by treatment with $1 \mu \mathrm{M}$ PF4778574 to the basal level of WT mice. Thus, administration of the brain-penetrable, highly selective AMPAR modulators could affect the excitatory neural transmission in the brain and lead the social behavioral changes. 
Then, how does the AMPAR activity modulation affect social behavior? We can speculate the answer from the function of AMPAR in the synapse. Since AMPARs mediate a majority of the rapid excitatory transmission in the synapse and precise control of neural synchrony [40-42], their functional problems possibly affect neural transmission and neural circuitry. In other words, increased and decreased AMPAR activity might cause noise and impaired connectivity, respectively, in the neural circuit due to altered excitatory neural activity. Indeed, VPA animal models display hyperconnectivity, hyper-reactivity, and hyper-plasticity, possibly due to enhanced excitatory neural transmission (reviewed in ref. [43]). On the other hand, a resting state functional magnetic resonance imaging (rsfMRI) study demonstrated that Cntnap2 KO mice exhibited reduced connectivity in the PFC and hippocampus. Additionally, these connectivity defects significantly correlated with reduced social behavior in Cntnap2 KO mice [44]. Thus, it is plausible that administration of AMPAR modulators rescues abnormal social behavior by normalizing hyper- or hypoexcitatory neural transmission, and thereby, correcting the abnormal neural connectivity in the respective mouse models. However, in case of Cntnap2 $\mathrm{KO}$ mice, there is another possibility regarding the effects of PF4778574 on improved social behaviors. A recent optogenetic study showed that enhancing the parvalbuminpositive (PV) interneuronal activity in the MPFC region rescued social deficits in Cntnap2 KO mice [45], indicating that suppressing neural activity in the $\mathrm{mPFC}$ region is required to rescue social deficits in Cntnap2 KO mice. Evidently, our results appear contradictory to those of Selimbeyoglu et al., but given that AMPARs are also observed in interneurons [46], and activate PV interneurons by feed-forward interaction (reviewed in ref. [47]), effects of the PF4778574 may not be limited to changes in excitatory neural transmission. Possibly, our systemic injection of PF4778574 activated both excitatory pyramidal neurons and PV interneurons, which might have rescued the decreased neuronal connectivity in the projections from the mPFC in Cntnap2 KO mice [44]. However, to address this possibility, further studies on the effects of regionand cell-type-specific AMPAR modulation on social behaviors of Cntnap2 KO mice will be required. In addition, to specify the role of AMPAR in regulating neural circuit connectivity, additional studies using $f M R I$ or EEG also will be required.

In our results, AMPAR activity modulation does not affect the repetitive or hyperactive behaviors, suggesting these behavioral changes may be independent of AMPAR activity. However, previous studies have suggested that NMDAR modulators can alleviate repetitive $[12,48,49]$ or hyperactive behaviors $[12,13$, 50], in addition to social behaviors, in ASD animal models. Moreover, abnormal function of NMDARs was implicated in the pathophysiology of repetitive or hyperactive behaviors [51, 52]. Given that NMDARs can regulate excitatory neural activity, $\mathrm{Ca}^{2+}$ influx, its related signaling pathways, and subsequently, synaptic plasticity (reviewed in ref. [53]), repetitive or hyperactive behavioral symptoms may have connection with NMDARmediated plasticity changes.

Here, we investigated the changes in glutamate receptor expression and function in the PFC or MPFC of both mouse models. The PFC is an important region for social recognition and emotional behavior not only in humans but also in rodents [5456]. In addition, neurological and functional defects in this region are frequently observed in patients with ASD or animal models $[56,57]$. Thus, investigating neurological or functional defects in the PFC is important to understand the pathophysiology of ASD. Although we could not elucidate the mPFC-specific role of AMPARs in regulating social behavior, our results demonstrate the clinical applicability of AMPAR modulators. Given that the route of administration in clinical studies is limited to the oral route, by which systemic exposure is inevitable, monitoring the effectiveness and toxicity of candidate drugs with a similar route of administration in preclinical studies is imperative.
Although several questions remain to be addressed due to the technical limitations, our findings illustrate the potential role of AMPARs in regulating social behavior, which enhances our understanding of the pathophysiology of ASD. Moreover, our findings suggest that classifying the patients with ASD depending on the intensity of $E / /$ imbalance may be a promising strategy to develop individualized therapeutics for these patients.

\section{ACKNOWLEDGEMENTS}

We are grateful to Dr. Carl Björkholm and Dr. Yong-Seok Lee for valuable and thoughtful comments on this manuscript. This work was supported by the National Research Foundation of Korea (NRF) grant funded by the Korea government (MSIP) (NRF-2014R1A2A2A01003079), and by a grant from the Korean Health Technology $R$ \& D Project through the Korea Health Industry Development Institute (KHIDI), funded by the Ministry of Health \& Welfare, Republic of Korea (No.: HI12C0021 0200).

\section{ADDITIONAL INFORMATION}

Supplementary Information accompanies this paper at (https://doi.org/10.1038/ s41386-018-0098-5).

Competing interests: The authors declare no competing interests.

Publisher's note: Springer Nature remains neutral with regard to jurisdictional claims in published maps and institutional affiliations.

\section{REFERENCES}

1. Lai MC, Lombardo MV, Baron-Cohen S. Autism. Lancet. 2014;383:896-910.

2. Abrahams BS, Arking DE, Campbell DB, Mefford HC, Morrow EM, Weiss LA, et al. SFARI Gene 2.0: a community-driven knowledgebase for the autism spectrum disorders (ASDs). Mol Autism. 2013;4:36.

3. Huguet $G$, Ey $E$, Bourgeron $T$. The genetic landscapes of autism spectrum disorders. Annu Rev Genom Hum Genet. 2013;14:191-213.

4. Nelson SB, Valakh V. Excitatory/inhibitory balance and circuit homeostasis in autism spectrum disorders. Neuron. 2015;87:684-98.

5. Won H, Mah W, Kim E. Autism spectrum disorder causes, mechanisms, and treatments: focus on neuronal synapses. Front Mol Neurosci. 2013;6:19.

6. Rubenstein JL, Merzenich MM. Model of autism: increased ratio of excitation/ inhibition in key neural systems. Genes Brain Behav. 2003;2:255-67.

7. Yizhar O, Fenno LE, Prigge M, Schneider F, Davidson TJ, O'Shea DJ, et al. Neocortical excitation/inhibition balance in information processing and social dysfunction. Nature. 2011;477:171-8.

8. Gao R, Penzes P. Common mechanisms of excitatory and inhibitory imbalance in schizophrenia and autism spectrum disorders. Curr Mol Med. 2015;15:146-67.

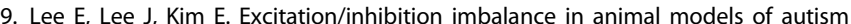
spectrum disorders. Biol Psychiatry. 2017;81:838-47.

10. Blatt GJ, Fatemi SH. Alterations in GABAergic biomarkers in the autism brain: research findings and clinical implications. Anat Rec. 2011;294:1646-52.

11. Tang G, Gudsnuk K, Kuo SH, Cotrina ML, Rosoklija G, Sosunov A, et al. Loss of mTOR-dependent macroautophagy causes autistic-like synaptic pruning deficits. Neuron. 2014;83:1131-43.

12. Kim JW, Seung H, Kim KC, Gonzales EL, Oh HA, Yang SM, et al. Agmatine rescues autistic behaviors in the valproic acid-induced animal model of autism. Neuropharmacology. 2017;113(Pt A):71-81.

13. Kim KC, Lee DK, Go HS, Kim P, Choi CS, Kim JW, et al. Pax6-dependent cortical glutamatergic neuronal differentiation regulates autism-like behavior in prenatally valproic acid-exposed rat offspring. Mol Neurobiol. 2014b;49:512-28.

14. Lee EJ, Choi SY, Kim E. NMDA receptor dysfunction in autism spectrum disorders. Curr Opin Pharmacol. 2015;20:8-13.

15. Won H, Lee HR, Gee HY, Mah W, Kim Jl, Lee J, et al. Autistic-like social behaviour in Shank2-mutant mice improved by restoring NMDA receptor function. Nature. 2012;486:261-5.

16. Purcell $A E$, Jeon $\mathrm{OH}$, Zimmerman AW, Blue $M E$, Pevsner J. Postmortem brain abnormalities of the glutamate neurotransmitter system in autism. Neurology. 2001:57:1618-28.

17. Marshall CR, Noor A, Vincent JB, Lionel AC, Feuk L, Skaug J, et al. Structural variation of chromosomes in autism spectrum disorder. Am J Hum Genet. 2008;82:477-88.

18. Pinto D, Pagnamenta AT, Klei L, Anney R, Merico D, Regan R, et al. Functional impact of global rare copy number variation in autism spectrum disorders. Nature. 2010;466:368-72. 
19. Ramanathan $S$, Woodroffe A, Flodman PL, Mays LZ, Hanouni M, Modahl CB, et al. A case of autism with an interstitial deletion on $4 q$ leading to hemizygosity for genes encoding for glutamine and glycine neurotransmitter receptor sub-units (AMPA 2, GLRA3, GLRB) and neuropeptide receptors NPY1R, NPY5R. BMC Med Genet. 2004;5:10.

20. Sanders SJ, Ercan-Sencicek AG, Hus V, Luo R, Murtha MT, Moreno-De-Luca D, et al. Multiple recurrent de novo CNVs, including duplications of the 7q11.23 Williams syndrome region, are strongly associated with autism. Neuron. 2011;70:863-85.

21. Wiedholz LM, Owens WA, Horton RE, Feyder M, Karlsson RM, Hefner K, et al. Mice lacking the AMPA GluR1 receptor exhibit striatal hyperdopaminergia and 'schizophrenia-related' behaviors. Mol Psychiatry. 2008;13:631-40.

22. Greer PL, Hanayama R, Bloodgood BL, Mardinly AR, Lipton DM, Flavell SW, et al. The Angelman syndrome protein Ube3A regulates synapse development by ubiquitinating arc. Cell. 2010;140:704-16.

23. Etherton MR, Tabuchi K, Sharma M, Ko J, Sudhof TC. An autism-associated point mutation in the neuroligin cytoplasmic tail selectively impairs AMPA receptormediated synaptic transmission in hippocampus. EMBO J. 2011:30:2908-19.

24. Alarcon M, Abrahams BS, Stone JL, Duvall JA, Perederiy JV, Bomar JM, et al. Linkage, association, and gene-expression analyses identify CNTNAP2 as an autism-susceptibility gene. Am J Hum Genet. 2008;82:150-9.

25. Strauss KA, Puffenberger EG, Huentelman MJ, Gottlieb S, Dobrin SE, Parod JM, et al. Recessive symptomatic focal epilepsy and mutant contactin-associated protein-like 2. N Engl J Med. 2006;354:1370-7.

26. Penagarikano O, Abrahams BS, Herman El, Winden KD, Gdalyahu A, Dong $H$, et al. Absence of CNTNAP2 leads to epilepsy, neuronal migration abnormalities, and core autism-related deficits. Cell. 2011:147:235-46.

27. Rasalam AD, Hailey H, Williams JH, Moore SJ, Turnpenny PD, Lloyd DJ, et al. Characteristics of fetal anticonvulsant syndrome associated autistic disorder. Dev Med Child Neurol. 2005;47:551-5.

28. Kim JW, Seung H, Kwon KJ, Ko MJ, Lee EJ, Oh HA, et al. Subchronic treatment of donepezil rescues impaired social, hyperactive, and stereotypic behavior in valproic acid-induced animal model of autism. PLoS ONE. 2014;9:e104927.

29. Roullet FI, Lai JK, Foster JA. In utero exposure to valproic acid and autism--a current review of clinical and animal studies. Neurotoxicol Teratol. 2013;36:47-56.

30. Rinaldi T, Kulangara K, Antoniello K, Markram H. Elevated NMDA receptor levels and enhanced postsynaptic long-term potentiation induced by prenatal exposure to valproic acid. Proc Natl Acad Sci USA. 2007;104:13501-6.

31. Menniti FS, Buchan AM, Chenard BL, Critchett DJ, Ganong AH, Guanowsky V, et al. CP-465,022, a selective noncompetitive AMPA receptor antagonist, blocks AMPA receptors but is not neuroprotective in vivo. Stroke. 2003;34:171-6.

32. Shaffer CL, Hurst RS, Scialis RJ, Osgood SM, Bryce DK, Hoffmann WE, et al. Positive allosteric modulation of AMPA receptors from efficacy to toxicity: The interspecies exposure-response continuum of the novel potentiator PF-4778574. J Pharmacol Exp Ther. 2013;347:212-24.

33. Rinaldi T, Perrodin C, Markram H. Hyper-connectivity and hyper-plasticity in the medial prefrontal cortex in the valproic Acid animal model of autism. Front Neural Circuits. 2008;2:4.

34. Varea O, Martin-de-Saavedra MD, Kopeikina KJ, Schurmann B, Fleming $\mathrm{HJ}$, Fawcett-Patel JM, et al. Synaptic abnormalities and cytoplasmic glutamate receptor aggregates in contactin associated protein-like 2/Caspr2 knockout neurons. Proc Natl Acad Sci USA. 2015;112:6176-81.

35. Anderson GR, Galfin T, Xu W, Aoto J, Malenka RC, Sudhof TC. Candidate autism gene screen identifies critical role for cell-adhesion molecule CASPR2 in dendritic arborization and spine development. Proc Natl Acad Sci USA. 2012;109:18120-5.

36. Gdalyahu A, Lazaro M, Penagarikano O, Golshani P, Trachtenberg JT, Geschwind $\mathrm{DH}$. The autism related protein contactin-associated protein-like 2 (CNTNAP2) stabilizes new spines: an in vivo mouse study. PLoS ONE. 2015;10:e0125633.
37. Kim KC, Kim P, Go HS, Choi CS, Park JH, Kim HJ, et al. Male-specific alteration in excitatory post-synaptic development and social interaction in pre-natal valproic acid exposure model of autism spectrum disorder. J Neurochem. 2013;124:832-43.

38. Lazzaro JT, Paternain AV, Lerma J, Chenard BL, Ewing FE, Huang J, et al. Functional characterization of CP-465,022, a selective, noncompetitive AMPA receptor antagonist. Neuropharmacology. 2002;42:143-53.

39. Balannik V, Menniti FS, Paternain AV, Lerma J, Stern-Bach Y. Molecular mechanism of AMPA receptor noncompetitive antagonism. Neuron. 2005;48:279-88.

40. Fricker D, Miles R. Interneurons, spike timing, and perception. Neuron 2001;32:771-4.

41. Jonas $P$, Bischofberger J, Fricker D, Miles R. Interneuron diversity series: Fast in fast out-temporal and spatial signal processing in hippocampal interneurons. Trends Neurosci. 2004;27:30-40.

42. Trussell LO. Synaptic mechanisms for coding timing in auditory neurons. Annu Rev Physiol. 1999;61:477-96.

43. Markram $\mathrm{K}$, Markram $\mathrm{H}$. The intense world theory-a unifying theory of the neurobiology of autism. Front Hum Neurosci. 2010;4:224.

44. Liska A, Bertero A, Gomolka R, Sabbioni M, Galbusera A, Barsotti N, et al. Homozygous loss of autism-risk gene CNTNAP2 results in reduced local and longrange prefrontal functional connectivity. Cereb Cortex. 2018;28:1141-53.

45. Selimbeyoglu A, Kim CK, Inoue M, Lee SY, Hong ASO, Kauvar I, et al. Modulation of prefrontal cortex excitation/inhibition balance rescues social behavior in CNTNAP2-deficient mice. Sci Transl Med. 2017;9:401.

46. Geiger JR, Melcher T, Koh DS, Sakmann B, Seeburg PH, Jonas $P$, et al. Relative abundance of subunit mRNAs determines gating and $\mathrm{Ca} 2+$ permeability of AMPA receptors in principal neurons and interneurons in rat CNS. Neuron. 1995;15:193-204.

47. Hu H, Gan J, Jonas P. Interneurons. Fast-spiking, parvalbumin(+) GABAergic interneurons: from cellular design to microcircuit function. Science. 2014;345:1255263.

48. Kang J, Kim E. Suppression of NMDA receptor function in mice prenatally exposed to valproic acid improves social deficits and repetitive behaviors. Front Mol Neurosci. 2015;8:17.

49. Presti MF, Mikes HM, Lewis MH. Selective blockade of spontaneous motor stereotypy via intrastriatal pharmacological manipulation. Pharmacol Biochem Behav. 2003;74:833-9.

50. Mohn AR, Gainetdinov RR, Caron MG, Koller BH. Mice with reduced NMDA receptor expression display behaviors related to schizophrenia. Cell. 1999;98:427-36.

51. Lewis M, Kim SJ. The pathophysiology of restricted repetitive behavior. J Neurodev Disord. 2009;1:114-32.

52. Archer T, Garcia D. Attention-deficit/hyperactivity disorder: focus upon aberrant N-methyl-D-aspartate receptors systems. Curr Top Behav Neurosci. 2016;29:295-311.

53. Luscher $C$, Malenka RC. NMDA receptor-dependent long-term potentiation and long-term depression (LTP/LTD). Cold Spring Harb Perspect Biol. 2012;4:a005777.

54. Etkin A, Egner T, Kalisch R. Emotional processing in anterior cingulate and medial prefrontal cortex. Trends Cogn Sci. 2011;15:85-93.

55. Forbes $\mathrm{CE}$, Grafman J. The role of the human prefrontal cortex in social cognition and moral judgment. Annu Rev Neurosci. 2010;33:299-324.

56. Bicks LK, Koike H, Akbarian S, Morishita H. Prefrontal cortex and social cognition in mouse and man. Front Psychol. 2015;6:1805.

57. Courchesne E, Mouton PR, Calhoun ME, Semendeferi K, Ahrens-Barbeau C, Hallet $M J$, et al. Neuron number and size in prefrontal cortex of children with autism. JAMA. 2011;306:2001-10. 